\title{
Multi-Sensor Optimal Data Fusion for INS/GPS/SAR Integrated Navigation System
}

\author{
Shesheng Gao ${ }^{\mathrm{a}}$, Yongmin Zhong ${ }^{\mathrm{b}}$, Xueyuan Zhang ${ }^{\mathrm{a}}$, Bijan Shirinzadeh ${ }^{\mathrm{c}}$ \\ ${ }^{a}$ School of Automation, Northwestern Polytechnical University, Xi'an 710072, China \\ ${ }^{\mathrm{b}}$ Department of Mechanical Engineering, Curtin University of Technology, Australia \\ ${ }^{\mathrm{c}}$ Department of Mechanical and Aerospace Engineering, Monash University, Australia
}

\begin{abstract}
INS/GPS/SAR integrated navigation system represents the trend of next generation navigation systems with the high performance of independence, high precision and reliability. This paper presents a new multi-sensor data fusion methodology for INS/GPS/SAR integrated navigation systems. This methodology combines local decentralized fusion with global optimal fusion to enhance the accuracy and reliability of integrated navigation systems. A decentralized estimation fusion method is established for individual integrations of GPS and SAR into INS to obtain the local optimal state estimations in a parallel manner. A global optimal estimation fusion theory is formulated to fuse the local optimal estimations for generating the global optimal state estimation of INS/GPS/SAR integrated navigation systems. The global data fusion features a method of variance upper finiteness and a method of variance upper bound to achieve the global optimal state estimation under a general condition. Experimental results demonstrate that INS/GPS/SAR integrated navigation systems developed by using the proposed methodology have a better performance than INS/GPS integrated systems.
\end{abstract}

Key words: integrated navigation system, data fusion, decentralized fusion and global optimal fusion. 


\section{Introduction}

INS (Inertial Navigation System) is a system that calculates the position, velocity, and attitude of a vehicle with the output of inertial sensors. The measurements of the inertial sensors contain errors due to physical limitations. These errors are accumulated in the navigation solution of INS, decreasing the accuracy of the solution. Therefore, if the error is not compensated with non-inertial sensors, the information of INS can only be trusted during a short period of time [12]. Nowadays, GPS (Global Positioning System) is commonly used as an aiding sensor in INS, and the INS/GPS integrated system has been widely applied to many navigation fields. However, GPS has a low sampling rate. It is also difficult to obtain continuous localization since the satellite signal may be lost and corrupted due to high buildings, tunnels and mountains, multi-path reflections and bad weather conditions $[4,10]$.

SAR (Synthetic Aperture Radar) is a new sensing technique by using active microwave imaging radar. It is an indispensable source of information in Earth observation since SAR is the only spaceborne sensor that has many advantages such as high resolution imaging, high penetration powder, far function range, wide survey band and all-weather working condition [9]. SAR overcomes the limitations of GPS, and the obtained high precision images can be used to correct the errors of INS according to the identified target information. On the other hand, INS provides the speed changing information, which can be used to compensate SAR since the changes of flight speed can disturb the imaging quality of SAR. Further, the precision of INS improved by the GPS information can also be used to compensate and adjust the SAR platform. This overcomes the poor imaging ability of SAR under some special circumstances such as air to air working condition, plain areas, the surface of water and 
deserts with faint terrain characters. From the above, it can be seen that the three systems INS, GPS and SAR complement each other. Due to the complementary nature of INS, GPS and SAR, INS/GPS/SAR integrated navigation system provides a promising solution for improving the performance of navigation.

The fundamental problem of integrated navigation systems is information fusion. The commonly used method is the centralized filter [2, 12], which provides the globally optimal state estimation by directly combining local measurement data. However, the centralized filter can cause a large computational burden in the fusion center due to the high-dimensional computation and large data memory [14]. Recently, decentralized information fusion has received attention, in which the information from local estimators yields the global optimal or suboptimal state estimation according to certain information fusion criteria. Various decentralized and parallel versions of the Kalman filter and their applications have been reported. One of the earliest decentralized filters is the federated square-root filter, in which a federated Kalman filter architecture was established for parallel processing [1]. Qiang and Harris discussed the functional equivalence of two measurement fusion methods, where the second method requires the measurement matrices to be of identical size [11]. Sun and Deng reported a three-layer optimal weighted fusion algorithm, which is weighted by matrices, diagonal matrices and scalars in the sense of linear minimum variance [14]. The optimal weighted fusion algorithm was further extended to smoothing fusion by using the fixed-interval Kalman filter [15]. Deng et al reported an optimal weighted fusion algorithm by using the steady-state Kalman filter [3]. The Bayesian algorithm [8] and the genetic algorithm [5] were also studied for decentralized fusion of multi-sensor data. In comparison with 
centralized data fusion, decentralized data fusion effectively utilizes information from a number of different sensors. It has many advantages such as lighter processing load, no requirement for a single centralized database, lower communication load, reduced possibility of data flow bottlenecks, easy fault detection and isolation, and high reliability [13]. However, the precision of decentralized fusion is generally lower than that of centralized fusion. In addition, most of the existing methods on decentralized fusion are unable to satisfy the requirement of real-time computation [3].

In general, most of the existing fusion methods for integrated navigation systems are mainly focused on the integration of INS and GPS. Further, data fusion architectures are mainly dominated by centralized fusion, especially in the military area [13]. In practical engineering applications, these methods suffer from either the low real-time performance due to the use of too many state variables or the low efficiency for fusion of multi-sensor information [6, 7].

This paper presents a new multi-sensor data fusion methodology for INS/GPS/SAR integrated navigation systems. It overcomes the disadvantages of decentralized fusion by combining local decentralized data fusion with global fusion into a two-level structure to improve the accuracy and reliability of integrated navigation systems. The dynamic model of INS/GPS/SAR integrated systems is developed to describe the system state and observation. A decentralized estimation fusion method is established for individual integrations of GPS and SAR into INS to independently obtain the local optimal state estimations of integrated subsystems INS/GPS and INS/SAR. A global optimal estimation fusion theory is formulated for fusion of the local optimal estimations to generate the global optimal state estimation of 
INS/GPS/SAR integrated navigation systems. The global data fusion features a method of variance upper finiteness and a method of variance upper bound to achieve the global optimal state estimation under a general condition. Experimental results are presented to demonstrate the efficacy of the proposed methodology.

\section{System Model}

SINS (Strap-down Inertial Navigation System) is used to establish the inertia navigation model. The navigation coordinate system of SINS is the E-N-U geography coordinate system. The state of the INS/GPS/SAR integrated navigation system is described by

$$
\dot{X}(t)=F(t) X(t)+G(t) \mathrm{W}(\mathrm{t})
$$

where $X(t)$ is the state vector of the system, $F(t)$ is the dynamic matrix of the system, $\mathrm{W}(\mathrm{t})$ is the system noise, and $G(t)$ is the noise coefficient matrix.

$X(t)$ is defined as

$$
\begin{gathered}
X(t)=\left[\delta v_{E}, \delta v_{N}, \delta v_{U}, \delta \varphi, \delta \lambda, \delta h, \Phi_{E}, \Phi_{N}, \Phi_{U}, \varepsilon_{X}, \varepsilon_{Y}, \varepsilon_{Z}, \varepsilon_{m x}, \varepsilon_{m y}, \varepsilon_{m z},\right. \\
\left.\nabla_{X}, \nabla_{Y}, \nabla_{Z}, \Delta t r, \delta \operatorname{tr}, \psi_{x}, \psi_{y}, \psi_{z}, \delta h_{b}\right]^{T}
\end{gathered}
$$

where $\left(\delta v_{E}, \delta v_{N}, \delta v_{U}\right)$ is the velocity error, $(\delta \varphi, \delta \lambda)$ is the position error, $\delta h$ is the altitude error, $\left(\Phi_{E}, \Phi_{N}, \Phi_{U}\right)$ is the attitude angle error, $\left(\varepsilon_{X}, \varepsilon_{Y}, \varepsilon_{Z}\right)$ is the gyro's constant drift, $\left(\varepsilon_{m x}, \varepsilon_{m y}, \varepsilon_{m z}\right)$ is the gyro's first-order Markov drift, $\left(\nabla_{X}, \nabla_{Y}, \nabla_{Z}\right)$ is the accelerator's zero offset, $\Delta t r$ and $\delta t r$ are the GPS clock bias and clock drift, $\left(\psi_{x}, \psi_{y}, \psi_{z}\right)$ is the SAR 
antenna's attitude angle error, and $\delta h_{b}$ is the barometric altimeter bias.

The observation information of the integrated INS/SAR/GPS system includes the GPS pseudo range $\rho_{G}$ and its rate $\dot{\rho}_{G}$, the INS pseudo range $\rho_{I}$ and its rate $\dot{\rho}_{I}$, the SAR azimuth angle $A$, and the SAR pitch angle $E$.

The system observation is described by

$$
Z_{K}=\left[\begin{array}{c}
\rho_{G}-\rho_{I} \\
\dot{\rho}_{G}-\dot{\rho}_{I} \\
A \\
E
\end{array}\right]+V_{K}
$$

By linearization, (3) becomes

$$
Z(t)=H(t) X(t)+V(t)
$$

where $H(t)$ and $V(t)$ are the observation matrix and observation noise of the integrated navigation system, respectively.

\section{Data Fusion Methodology}

An optimal estimation fusion methodology is established to efficiently integrate the subsystems GPS and SAR into the main system INS. As shown in Fig. 1, this methodology combines local decentralized fusion with global optimal fusion into a two-level structure to achieve the accuracy and reliability of the integrated system from the overall view of point. In the first level, subsystems SAR and GPS are integrated with INS by two local filters (Local 
Filter 1 and Local Filter 2) respectively to obtain the local optimal state estimations. This level is a parallel process of decentralized estimation based on the observation space. In the second level, the local optimal estimations generated from these two local filters are fused together by the global filter to generate the global optimal state estimation of the INS/GPS/SAR integrated navigation system.

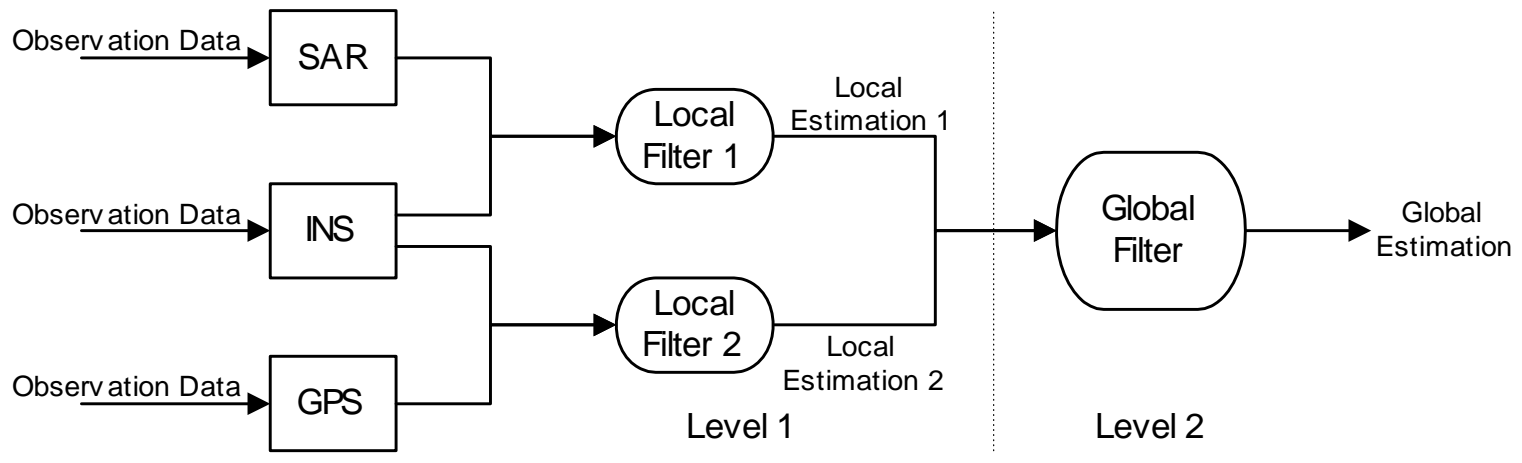

Figure 1. Framework of the multi-sensor data fusion

\subsection{Decentralized Estimation Fusion}

The state of INS is described by

$$
x(k+1)=\Phi(k) x(k)+G(k) w(k)
$$

where $x(k) \in R^{n}$ is the system state, $\Phi(k)$ is the transition matrix of the system state, $G(k)$ is a given matrix, and $w(k)$ is the Gauss white noise with zero mean and variance $Q(k) \geq 0$, i.e. $E\{w(k)\}=0$ and $E\left\{w(k) w^{T}(k)\right\}=Q(k)$.

Subsystems SAR and GPS independently observe the output of INS. The two local filters estimate the state of INS according to the observed data. The model of the $i$ th $(i=1,2)$ local filter is described by the following equations 


$$
\begin{gathered}
x_{i}(k+1)=\Phi(k) x_{i}(k)+G(k) w(k) \\
y_{i}(k)=H_{i}(k) x_{i}(k)+v_{i}(k)
\end{gathered}
$$

where $y_{i}(k) \in R_{i}^{m}$ is the output of the $i$ th subsystem, and $w(k)$ and $v_{i}(k)$ are the white noises that are independent of one another and have zero mean, i.e. $E\left\{v_{i}(k) v_{j}^{T}(k)\right\}=R_{i j}(k) \delta_{i j}$ and $E\left\{v_{i}(k)\right\}=0$.

Each local filter calculates the optimal estimation of the local state $x_{i}(k)$ by using the Kalman filter. The Kalman filtering algorithm for the $i$ th $(i=1,2)$ local filter is described by the following equations

$$
\begin{gathered}
\hat{x}_{i}(k+1 \mid k+1)=\hat{x}_{i}(k+1 \mid k)+K_{i}(k+1)\left[y_{i}(k+1)-H_{i}(k+1) x_{i}(k+1 \mid k)\right] \\
\hat{x}_{i}(k+1 \mid k)=\Phi(k) \hat{x}_{i}(k \mid k) \\
K_{i}(k+1)=P_{i}(k+1 \mid k) H_{i}^{T}(k+1)\left[H_{i}(k+1) P_{i}(k+1 \mid k) H_{i}^{T}(k+1)+R_{i}(k+1)\right]^{-1} \\
P_{i}(k+1 \mid k)=\Phi(k) P_{i}(k \mid k) \Phi^{T}(k)+G(k) Q(k) G^{T}(k) \\
P_{i}(k+1 \mid k+1)=\left[I-K_{i}(k+1) H_{i}(k+1)\right] P_{i}(k+1 \mid k)
\end{gathered}
$$

After completing the computations of the two decentralized parallel-processing local filters, two local optimal state estimations $\hat{x}_{1}(k)$ and $\hat{x}_{2}(k)$ can be obtained and further be fused by the global filter.

\subsection{Global Optimal Estimation Fusion}

Suppose that the state estimations of Local Filters 1 and 2 are $\hat{x}_{1}$ and $\hat{x}_{2}$, and the error variances corresponding to $\hat{x}_{1}$ and $\hat{x}_{2}$ are $\hat{p}_{11}$ and $\hat{p}_{12}$, respectively. If local estimations 
$\hat{x}_{1}$ and $\hat{x}_{2}$ are independent of one another, the following Lemma can be obtained.

Lemma 1 For a linear discrete system, assume that the state estimation obtained by the ith local filter is $\hat{x}_{i}$ and the corresponding error variances are $p_{i i}(i=1,2)$, and $p_{i j}=0 \quad(i, j=1,2$ and $i \neq j)$. Then, the global optimal state estimation may be written as

$$
\hat{x}_{g}=P_{g} \sum_{i=1}^{2} p_{i i}^{-1} \hat{x}_{i}
$$

where

$$
P_{g}=\left(\sum_{i=1}^{2} p_{i i}^{-1}\right)^{-1}
$$

The above Lemma can be rigorously proved by using mathematical induction (see Appendix).

It is noted that (13) holds only under the condition that $\hat{x}_{1}$ and $\hat{x}_{2}$ are independent of one another. To extend (13) to a more general condition, a method of variance upper finiteness is established to improve Lemma 1.

Assume that the local state is $x_{i}(i=1,2)$, the variance of the estimation error is $p_{i i}$, and the covariance is $p_{i j}(i \neq j)$. Further, define an augmented state vector as $x=\left[x_{1}^{T}, x_{2}^{T}\right]$. Then, the augmented state and variance are described by

$$
\left[\begin{array}{l}
\dot{x}_{1} \\
\dot{x}_{2}
\end{array}\right]=\operatorname{diag}[\phi \quad \phi]\left[\begin{array}{l}
x_{1} \\
x_{2}
\end{array}\right]+\left[\begin{array}{l}
G \\
G
\end{array}\right] w(k)
$$

and 


$$
P=\left[\begin{array}{ll}
p_{11} & p_{12} \\
p_{21} & p_{22}
\end{array}\right]
$$

where $\phi$ is the state transition matrix, $G$ is a given matrix, and $w(k)$ is the Gauss white noise with zero mean and $Q(k) \geq 0$ variance, i.e. $E(w(k)]=0$ and $E\left[w(k) w^{\mathrm{T}}(k)\right]=Q(k)$. The local observation of the $i$ th subsystem is

$$
y_{i}=H_{i} x_{i}+v_{i}=H X+v_{i} \quad(i=1,2)
$$

where $v_{i}$ is the white noise that is independent of $w(k)$ and has zero mean and variance $R_{i}$, and $H$ is the observation matrix.

Let

$$
A=H P H^{T}+R_{i}=H_{i} P_{i i} H_{i}^{T}+R_{i}
$$

By using the Kalman filter algorithm, the observation of the state estimation can be updated as

$$
\hat{x}^{*}=\hat{x}+P H^{T} A^{-1}(y-H \hat{x})
$$

where $\hat{X}^{*}$ is the new observation of the state estimation.

The $j$ th component of the observation is 


$$
\hat{x}_{j}^{*}=\hat{x}_{j}+p_{j i} H_{i}^{T} A^{-1}\left(y_{i}-H_{i} X_{i}\right)
$$

Further, we have

$$
P^{*}=P-K H P=P-P H^{T} A^{-1} H P \quad\left(K=P H^{T} A^{-1}\right)
$$

The $j k$ th partitioning element of $P^{*}$ is

$$
p_{j k}{ }^{*}=p_{j i} H_{i}^{T} A^{-1} H_{i} p_{k i}^{T}
$$

By analyzing (20)-(22), the following remarks can be obtained.

Remark 1 When $j=i,(20)$ becomes

$$
x_{i}^{*}=\hat{x}_{i}+p_{i i} H_{i}^{T} A^{-1}\left(y_{i}-H_{i} \hat{x}_{i}\right)
$$

which means $y_{i}$ only affects the local state $x_{i}$.

Remark 2 When $j \neq i$ and $p_{i j}(0)=0$, there is $p_{i j}^{*}(k)=0$ by (22); and when $j=i$, there is $p_{i i}^{*}=p_{i i} H-p_{i i} H_{i}^{T} A^{-1} H_{i} p_{i i}^{T}$.

Remark 3 When $j \neq i$ and $p_{i j}=0$, it can be seen from (20) that observation $y_{i}$ does not affect $\hat{x}_{j}$, and thus $\hat{x}_{i}^{*}=\hat{x}_{i}$.

It can be seen from the above remarks that if the local state estimations are not dependent of each other initially $\left(p_{i j}(0)=0\right)$, each of the local filters does not affect each other during 
the process of observation update, and thus filtering can be performed in a decentralized and parallel manner. Subsequently, the dynamic update equations are

$$
\left[\begin{array}{l}
\hat{x}_{1} \\
\hat{x}_{2}
\end{array}\right]=\operatorname{diag}\left[\begin{array}{ll}
\phi & \phi
\end{array}\right]\left[\begin{array}{l}
\hat{x}_{1}^{*} \\
\hat{x}_{2}^{*}
\end{array}\right]
$$

and

$$
\left[\begin{array}{ll}
p_{11} & p_{12} \\
p_{21} & p_{22}
\end{array}\right]=\operatorname{diag}\left[\begin{array}{ll}
\phi & \phi
\end{array}\right]\left[\begin{array}{ll}
p_{11}^{*} & p_{12}^{*} \\
p_{21}^{*} & p_{22}^{*}
\end{array}\right] \operatorname{diag}\left[\begin{array}{ll}
\phi & \phi
\end{array}\right]+\left[\begin{array}{l}
G \\
G
\end{array}\right] Q\left[\begin{array}{ll}
G^{T} & G^{T}
\end{array}\right]
$$

It can be seen from (24) and (25) that (i) the state estimation is separate; and (ii) if there is no system noise (i.e. $Q=0)$ and the variance of initial estimations is $p_{i j}(0)=0$, there is $p_{j i}(k)=0$.

Remark 4 For a linear discrete system, suppose that

(i) the state estimation obtained by the ith local filter is $\hat{x}_{i}$;

(ii) the corresponding estimation variance is $p_{i i}(i=1,2)$; and

(iii) $p_{i j}(0)=0 \quad(i \neq j)$ and $Q=0$.

Then, the conclusion of Lemma 1 remains true.

Although Remark 4 relaxes the condition of Lemma 1 towards a more general condition, it still requires that the system have no noise. This condition is difficult to be satisfied in practical engineering applications. Therefore, a method of variance upper bound is established to deal with the case when the system has noise. 
When there is noise in the system, it can be seen from (25) that $p_{i j}(k)=0(i \neq j)$ cannot be guaranteed even if $p_{i j}(0)=0$. Consider

$$
\left[\begin{array}{l}
G \\
G
\end{array}\right]\left[\begin{array}{ll}
G^{T} & G^{T}
\end{array}\right]=\left[\begin{array}{ll}
G & \\
& G
\end{array}\right]\left[\begin{array}{ll}
Q & Q \\
Q & Q
\end{array}\right]\left[\begin{array}{ll}
G^{T} & \\
& G^{T}
\end{array}\right]
$$

By the matrix theory, it is known that a $N \times N$ matrix composed of element $Q$ has an upper bound

$$
\left[\begin{array}{ccc}
Q & \cdots & Q \\
\vdots & \ddots & \vdots \\
Q & \cdots & Q
\end{array}\right]_{N \times N} \leq\left[\begin{array}{ccc}
r_{1} Q & & \\
& \ddots & \\
& & r_{N} Q
\end{array}\right]_{N \times N}
$$

where $1 / r_{1}+\cdots+1 / r_{N}=1,1 \leq r_{i} \leq \infty$ and $i=1,2, \cdots, N$.

By (27), when $N=2$, we have

$$
\left[\begin{array}{ll}
Q & Q \\
Q & Q
\end{array}\right] \leq\left[\begin{array}{ll}
r_{1} Q & \\
& r_{2} Q
\end{array}\right]
$$

Replacing (28) into (25), there is

$$
\left[\begin{array}{ll}
p_{11} & p_{12} \\
p_{21} & p_{22}
\end{array}\right] \leq\left[\begin{array}{ll}
\phi & \\
& \phi
\end{array}\right]\left[\begin{array}{ll}
p_{11}^{*} & p_{12}^{*} \\
p_{21}^{*} & p_{22}^{*}
\end{array}\right]\left[\begin{array}{ll}
\phi^{T} & \\
& \phi^{T}
\end{array}\right]+\left[\begin{array}{ll}
G & \\
& G
\end{array}\right]\left[\begin{array}{ll}
r_{1} Q & \\
& r_{2} Q
\end{array}\right]\left[\begin{array}{ll}
G^{T} & \\
& G^{T}
\end{array}\right]
$$

After replacing the variance matrix with the upper bound of the variance matrix, the right side 
of (29) is actually the addition of two diagonal matrices. If $p_{i j}=0(i \neq j)$, there is $p_{i j}(k)=0(i \neq j)$ after simple computations. Therefore, the separate dynamic update equations of the estimation error variance can be obtained as

$$
\begin{aligned}
& p_{i j}=\phi p_{i i}^{*}+r_{i} G Q G^{T} \quad(j=1,2) \\
& p_{i j}=\phi p_{i j}^{*} \phi^{T}
\end{aligned}
$$

Accordingly, (13)、(14)、(24)、(30) and (31) are the formulas of the global optimal estimation fusion under the general condition. The global optimal estimation fusion can also be extended to the optimal data fusion of an integrated navigation system consisted of several subsystems.

\section{Experimental Analysis and Discussions}

A prototype system has been developed for INS/GPS/SAR integrated navigation by using the proposed methodology. This system fuses the information from integrated subsystems INS/GPS and INS/SAR to achieve navigation. Simulation experiments have been conducted to verify the performance of the proposed data fusion methodology.

The initial position of the airplane is at East longitude $120^{\circ}$, North latitude $30^{\circ}$ and altitude $1000 \mathrm{~m}$. The flight direction is to the East, the flight speed is $300 \mathrm{~m} / \mathrm{s}$, and the flight time is 1000s. The Gyro drift is $0.1^{\circ} / h$, the white noise is $0.001^{\circ} / h$, and the related time is $1 \mathrm{~h}$. The accelerator's zero offset is $10^{-4} \mathrm{~g}$, the white noise is $10^{-5} \mathrm{~g}$, and the related time is 0.5h. The initial navigation information is given an $1000 "$ altitude angle error, a $30 \mathrm{~m}$ 
position error, and a $0.01 \mathrm{~m} / \mathrm{s}$ velocity error. Fig. 2 and Fig. 3 illustrate the position errors, velocity errors and attitude errors of the INS/GPS and INS/GPS/SAR integrated navigation systems under the condition of white noise, respectively.
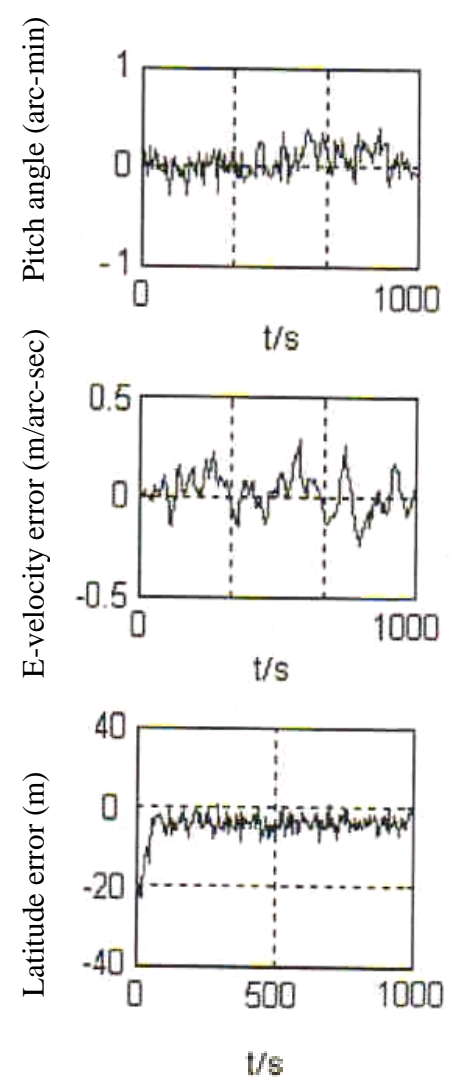
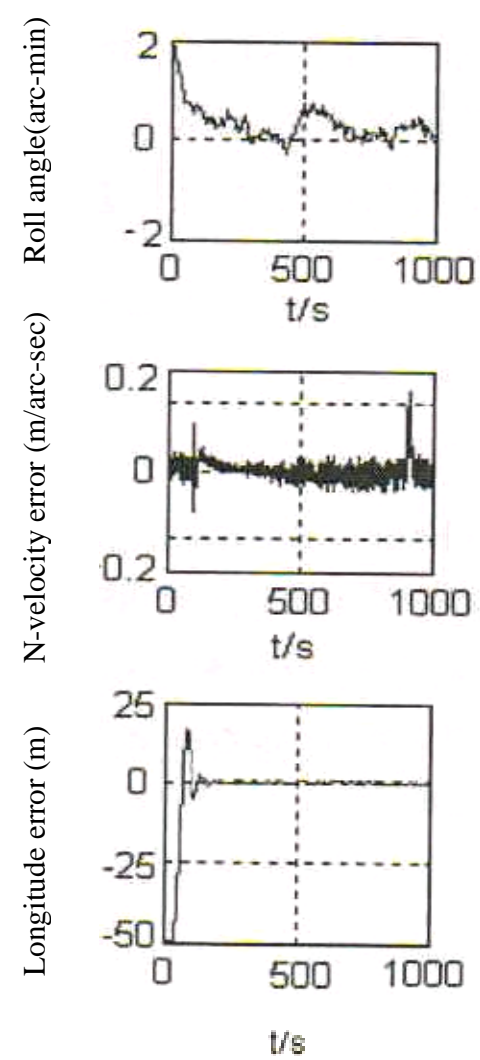
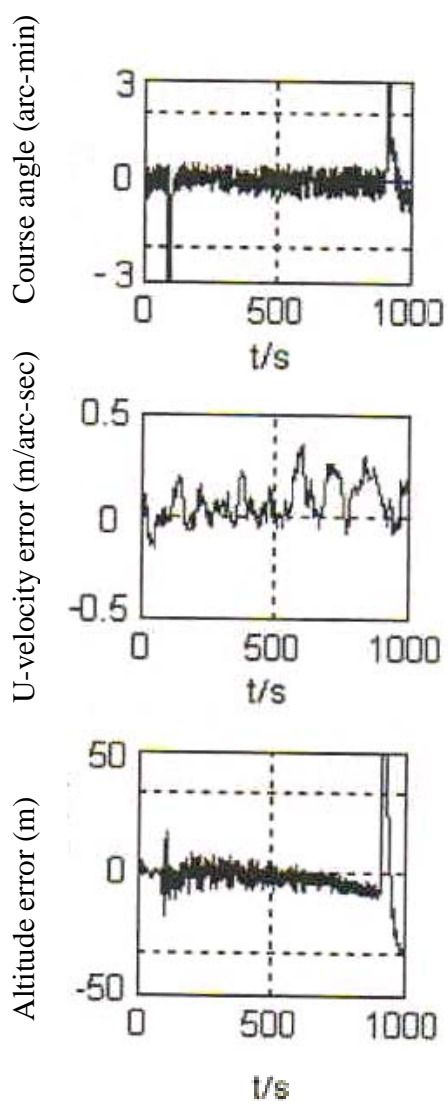

Figure 2. Filter curves of the INS/GPS integrated navigation system

Comparing Fig. 2 with Fig. 3, it can be seen that the position errors and velocity errors in these two systems decrease gradually with respect to time. However, the errors of the INS/SAR/GPS integrated navigation system are smaller than those of the INS/GPS integrated navigation system. Especially, the altitude error of the INS/SAR/GPS integrated navigation system is smaller than that of the INS/GPS integrated navigation system, which is almost divergent after the time point of 850s. In the INS/SAR/GPS integrated navigation system, all of the position errors and velocity errors reach the steady state within a very short time, and 
remain stably at the very low velocity errors. At the time points around 850s, the attitude errors and course angle errors of the INS/GPS integrated navigation system are larger than those of the INS/SAR/GPS integrated navigation system. It is also obvious that the velocity errors of the INS/SAR/GPS integrated navigation system are smaller than those of the INS/GPS integrated navigation system. From the simulation results, it can be seen that the proposed data fusion methodology can greatly improve the accuracy of integrated navigation systems. Further, the INS/GPS/SAR integrated system developed by using the proposed fusion methodology has a better performance than the INS/GPS integrated system.
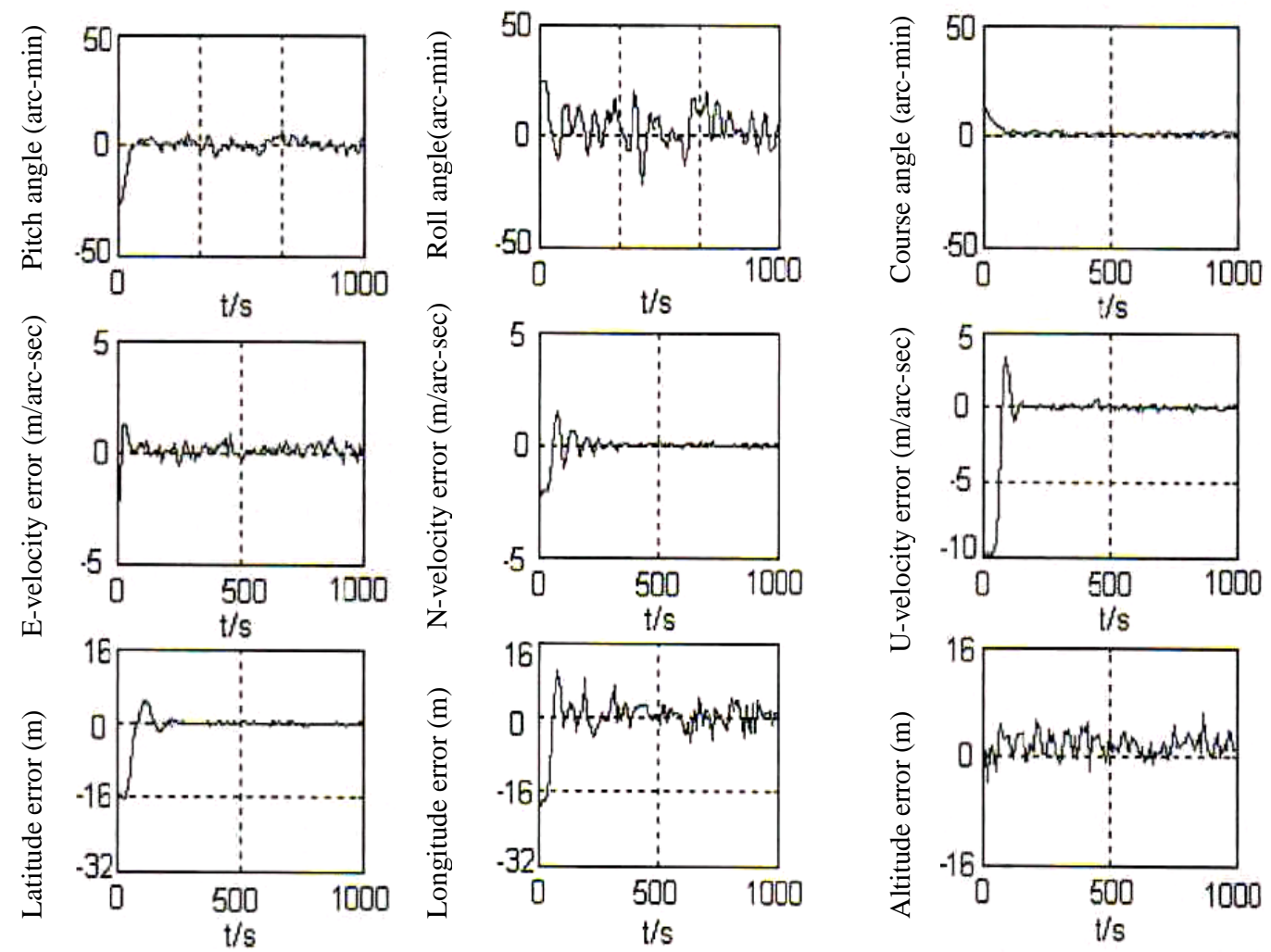

Figure 3. Filter curves of the proposed INS/ GPS/SAR integrated navigation system 


\section{Conclusions}

This paper presents a new multi-sensor data fusion methodology for INS/GPS/SAR integrated navigation systems. This methodology combines local decentralized fusion with global estimation fusion into a two-level structure to enhance the accuracy and reliability of integrated navigation systems. A decentralized estimation fusion method is established for individual integrations of GPS and SAR into INS to independently obtain the local optimal state estimations. A global optimal estimation fusion theory is studied to fuse the local optimal estimations for generating the global optimal state estimation of INS/GPS/SAR integrated navigation systems. The methodology overcomes the shortcomings of decentralized fusion and fully takes advantage of each subsystem's navigational information to increase the accuracy and fault tolerance of integrated navigation systems. The resultant INS/GPS/SAR integrated navigation systems have a better performance than INS/GPS integrated systems.

Future research work is mainly focused on establishment of intelligent fusion methodologies for INS/GPS/SAR integrated navigation systems. Advanced expert systems and neural networks, together with the emerging random weighting method for optimal estimation [16, 17] will be established to automatically and optimally fuse the information from INS, GPS and SAR for further improvement of integrated INS/GPS/SAR navigation systems.

\section{Appendix Proof of Lemma 1}

Proof. When $n=2$, it is noted $p_{i j}=0 \quad(i, j=1,2$ and $i \neq j)$. Therefore, $\hat{x}_{1}$ and $\hat{x}_{2}$ are 
independent of each other. Subsequently, we have

$$
\hat{x}_{g}=\left(p_{11}^{-1}-p_{22}^{-1}\right)\left(p_{11}^{-1} \hat{x}_{1}+p_{22}^{-1} \hat{x}_{2}\right)
$$

and

$$
\hat{p}_{g}=\left(p_{11}^{-1}-p_{22}^{-1}\right)^{-1}
$$

Suppose that (13) holds when $n=N-1$. Then, the global state estimation $\hat{x}_{g}^{N-1}$ may be written as

$$
\hat{x}_{g}^{N-1}=p_{g}^{N-1} \sum_{i=1}^{n} p_{i i}^{-1} \hat{x}_{i}
$$

When $n=N$, by the similar derivation, we have

$$
\hat{x}_{g}=p_{g}\left[\left(p_{g}^{N-1}\right)^{-1} \cdot \hat{x}_{g}^{N-1}+p_{N N}^{-1} \hat{X}_{N}\right]
$$

and

$$
p_{g}=\left[\left(p_{g}^{N-1}\right)^{-1}+p_{N N}^{-1}\right]^{-1}
$$

Therefore, we can obtain 


$$
p_{g}=\left[\sum_{i=1}^{n} p_{i i}^{-1}+p_{N N}^{-1}\right]^{-1}=\left(\sum_{i=1}^{n} p_{i i}^{-1}\right)^{-1}
$$

and

$$
\hat{x}_{g}=p_{g}\left[\sum_{i=1}^{n} p_{i i}^{-1} \hat{x}_{i}+p_{N N}^{-1} \hat{x}_{N}\right]=p_{g} \sum_{i=1}^{n} p_{i i}^{-1} \hat{x}_{i}
$$

\section{References}

1. N. A. Carlson, Federated square root filter for decentralized parallel processes, IEEE Transactions on Aerospace and Electronic Systems, Vol. 26, No. 3, 1990, pp517-525.

2. F. Caron, E. Duflos, D. Pomorski, P. Vanheeghe, GPS/IMU data fusion using multisensor Kalman filtering: introduction of contextual aspects. Information Fusion, Vol. 7, No. 2, 2006, pp221-230.

3. Z.-L. Deng, Y. Gao, L. Mao, Y. Li, G. Hao, New approach to information fusion steady-state Kalman filtering, Automatica, Vol. 41, No. 10, 2005, pp1695-1707.

4. J. Farrell, The Global Positioning System and Inertial Navigation, McGraw-Hill professional, New York, 1998.

5. N. Gnanapandithan, B. Natarajan, Joint Optimization of Local and Fusion Rules in a Decentralized Sensor Network, Journal of Communications, Vol. 1, No. 6, 2006, pp9-17.

6. R. Kumar, M. Wolenetz, B. Agarwalla, A framework for distributed data fusion, Information Fusion, Vol. 8, No. 3, 2007, pp227-251.

7. J. Ma, J. Zhang, J. Yang, N. Zhang, An improved information fusion algorithm based SVM, IEEE International Conference on Computational Intelligence and Security Workshops, Harbin, China, 2007, pp397-400.

8. A. Makarenko, H. Durrant-Whyte, Decentralized Bayesian algorithms for active sensor networks, Information Fusion, Vol. 7, No. 4, 2006, pp418-433.

9. A. Moreira, D. Hounam, W. Wiesbeck, Foreword to the Special Issue on Synthetic Aperture Radar (SAR) Technologies and Techniques, IEEE Transactions on Geoscience and Remote Sensing, Vol. 45, No. 11, 2007, pp3303-3305.

10. A. Noureldin, A. El-Shafie, M. R. Taha, Optimizing neuro-fuzzy modules for data fusion of vehicular navigation systems using temporal cross-validation, Engineering Applications of Artificial Intelligence, Vol. 20, No. 1, 2007, pp49-61.

11. G. Qiang, C. J. Harris, Comparison of two measurement fusion methods for Kalman-filter-based multisensor data fusion, IEEE Transactions on Aerospace and 
Electronic Systems, Vol. 37, No. 1, 2001, pp273-280.

12. J. Seo, J. G. Lee, Application of nonlinear smoothing to integrated GPS/INS navigation system, Journal of Global Positioning Systems, Vol. 4, No. 2, 2005, pp88-94.

13. D. Smith, S. Singh, Approaches to multisensor data fusion in target tracking: a survey, IEEE Transactions on Knowledge and Data Engineering, Vol. 18, No. 12, 2006, pp1696-1710.

14. S.-L. Sun, Z.-L. Deng, Multi-sensor optimal information fusion Kalman filter, Automatics, Vol. 40, No. 6, 2004, pp1017-1023.

15. S.-L. Sun, Multi-sensor optimal fusion fixed-interval Kalman smoothers, Information Fusion, Vol. 9, No. 2, 2008, pp293-299.

16. S. Gao, Z. Feng, Y. Zhong and B. Shirinzadeh, Random Weighting Estimation of Parameters in Generalized Gaussian Distribution, Information Sciences, Vol. 178, No. 9, 2008, pp2275-2281.

17. Gao, S., Zhang, Z., Yang, B., 2004. The random weighting estimation of quantile process. Information Sciences 164(1-4), 139-146. 